\title{
Estudio de las lesiones neoplásicas metacrónicas en el carcinoma colorrectal
}

\author{
Study of colorectal metachronous neoplastic lesions
}

\author{
A. Borda ${ }^{1}$, M. Muñoz-Navas ${ }^{2}$, J.M. Martínez-Peñuela ${ }^{1}$, F.J. Jiménez ${ }^{1}$, C. Carretero ${ }^{2}$, \\ F. Borda ${ }^{1}$
}

\section{RESUMEN}

Fundamento. Analizar la frecuencia y las características de las lesiones neoplásicas metacrónicas, carcinomas y adenomas, tras la resección de un cáncer colo-rectal (CCR).

Pacientes y métodos. Revisamos 382 CCR operados y seguidos mediante colonoscopias completas en dos hospitales de nuestra comunidad. Analizamos las lesiones metacrónicas registradas valorando su localización, momento del diagnóstico, histología, número y tamaño. Estudiamos la frecuencia de adenomas de aparición precoz (12 meses), comparando su tamaño con respecto al resto de lesiones.

Resultados. La mediana de seguimiento fue de 48 meses (12112), con 2,74 $\pm 1,47$ colonoscopias/caso. Diagnosticamos 7 cánceres metacrónicos $(1,8 \%), 4$ de ellos en estadio I. La mediana de tiempo hasta su diagnóstico fue de 24 meses (13-54). Registramos adenomas metacrónicos en 162 casos (42,4\%), sin diferencias entre los dos hospitales: $42,1 \%$ vs. $43,8 \%$ $(\mathrm{p}=0,88)$. Un $6,3 \%$ de los pacientes presentaron adenomas avanzados. En 164 casos en que el primer control se efectuó a los 12 meses, la incidencia de adenomas fue del $24 \%$. Los adenomas fueron mayoritariamente únicos $(60,8 \%)$ y menores de $5 \mathrm{~mm}$ (68,5\%). En un 55,5\% de los casos con pólipos, alguno tenía una localización proximal. El diagnóstico se realizó en la $1^{\text {a }}$ exploración $(56,2 \%), 2^{\mathrm{a}}(27,8 \%)$ ó $3^{\mathrm{a}}(9 \%)$. La mediana de tiempo hasta el diagnóstico fue de 21 meses (12-112) para el adenoma simple y de 35 (12-112) para el avanzado.

Conclusiones. Nuestro seguimiento permitió aplicar un tratamiento teóricamente curativo en la mayoría de los carcinomas metacrónicos diagnosticados. La alta incidencia de adenomas y su frecuente localización proximal hacen necesario un seguimiento con colonoscopias completas, que debería iniciarse al año de la operación y podría pasar a ser menos estricto tras tres exploraciones consecutivas sin pólipos.

Palabras clave. Cáncer colo-rectal. Carcinoma metacrónico. Adenoma metacrónico.

\begin{abstract}
Background. To analyse the frequency and characteristics of metachronous neoplastic lesions, carcinomas and adenomas, following resection of colorectal cancer.

Patients and methods. We reviewed 382 patients subjected to CCR operations and followed up through complete colonoscopies in two hospitals in our province. We analysed the metachronous lesions registered, evaluating their localisation, time of diagnosis, histology, number and size. We studied the frequency of early adenomas (12 months), comparing their size with the rest of the lesions.
\end{abstract}

Results. The average follow-up was 48 months (12-112), with $2.74 \pm 1.47$ colonoscopies/case. We diagnosed 7 metachronous cancers $(1.8 \%), 4$ of them in stage I. The average time until their diagnosis was 24 months (13-54). We registered metachronous adenomas in 162 cases (42.4\%), without differences between the two hospitals: $42.1 \%$ vs. $43.8 \%(\mathrm{p}=0.88)$. Six point three percent of the patients presented advanced adenomas. In 164 cases where the control was carried out after 12 months, the incidence of adenomas was $24 \%$. In the majority of cases, the adenomas were sole $(60.8 \%)$ and smaller than 5 $\mathrm{mm}(68.5 \%)$. In $55.5 \%$ of the cases with polyps, some had a proximal localisation. Diagnosis was made on the $1^{\text {st }}$ exploration $(56.2 \%)$, the $2^{\text {nd }}(27.8 \%)$ or the $3^{\text {rd }}(9 \%)$. Average time until diagnosis was 21 months (12-112) for simple adenoma and 35 (12-112) for advanced adenoma.

Conclusions. Our follow up made it possible to apply a theoretically curative treatment in the majority of the metachronous carcinomas diagnosed. The high incidence of adenomas and the frequent proximal localisation make a follow up with complete colonoscopies necessary, which must be started one year after the surgery and can become less strict following three consecutive explorations without polyps.

Key words. Colorectal cancer. Metachronous carcinoma. Metachronous adenoma.
1. Servicios de Digestivo y Anatomía Patológica. Hospital de Navarra. Pamplona.

2. Departamento de Digestivo. Clínica Universidad de Navarra. Pamplona.

Recepción: 20 de julio de 2009

Aceptación provisional: 18 de septiembre de 2009

Aceptación definitiva: 7 de octubre de 2009

\section{Correspondencia}

Ana Borda Martín

Olite $37-7^{\circ}$

31004 Pamplona

Tfno. 848422113

E-mail: abordamartin@yahoo.es 


\section{INTRODUCCIÓN}

El cáncer colo-rectal (CCR) se ha venido considerando como el segundo en frecuencia dentro de los países desarrollados ${ }^{1}$, en los que se ha estimado una posibilidad de presentarlo a lo largo de la vida de un $5 \%^{2}$, ocupando en España el segundo puesto en cuanto a número de muertes ocasionadas por cáncer ${ }^{3}$, tanto en hombres como en mujeres. Según las cifras de la base de datos GLOBOCAN 2000, elaborada por la Internacional Agency Research Cancer (IARC; http://www-dep.iarc.fr), el CCR ha pasado a ser el tumor más diagnosticado en Europa, con 304.687 casos nuevos/año, habiendo superado al cáncer de pulmón: 301.090 casos/año. En España las estimaciones son de 25.000 nuevos diagnósticos y de 12.500 fallecimientos/año ${ }^{4}$.

Todos estos datos confirman la gran trascendencia del carcinoma de colon y recto en nuestro medio, con importantes consecuencias sanitarias y económicas. La gravedad del proceso, unida a los elevados costes de su diagnóstico, tratamiento y seguimiento elevan al CCR a la categoría de problema socio-sanitario de primer orden.

La gran mayoría de los CCR se desarrollan sobre un adenoma previo, siguiendo una secuencia multipasos adenoma-carcinoma $^{1,4,5}$ y siendo muy frecuente la presencia de lesiones sincrónicas, adenomas o menos habitualmente carcinomas, en el momento del diagnóstico ${ }^{6,7}$.

Una vez efectuada la extirpación endoscópica de las posibles lesiones sincrónicas y la resección quirúrgica del tumor, la multicentricidad tumoral puede ocasionar el desarrollo de nuevas lesiones neoplásicas, denominadas metacrónicas. $\mathrm{Al}$ igual que las lesiones sincrónicas, las metacrónicas serán en su mayor parte adenomas y con mucha menor frecuencia carcinomas colo-rectales. Un correcto diagnóstico de las posibles lesiones neoplásicas metacrónicas es importante para poder aplicar un tratamiento con mayores posibilidades curativas, tanto quirúrgico como de resección endoscópica en el caso de los adenomas.
La incidencia de lesiones metacrónicas presenta importantes variaciones en los diferentes trabajos publicados ${ }^{8-12}$, siendo muy escasos los datos referentes a Espa$\tilde{n} \mathrm{a}^{13} \mathrm{y}$ careciendo de ellos en nuestra comunidad.

El objetivo del trabajo ha sido analizar en nuestro medio la frecuencia, momento del diagnóstico y características de las lesiones metacrónicas que se presentan tras la cirugía, efectuada con intención curativa, en el CCR.

\section{PACIENTES Y MÉTODOS}

Analizamos retrospectivamente 382 pacientes con carcinomas de colon o recto, diagnosticados mediante colonoscopia completa pre, intra o post-operatoria en dos hospitales de Pamplona. En todos los pacientes se efectuó una resección quirúrgico-endoscópica de las lesiones neoplásicas iniciales, estudio anatomopatológico de la pieza y seguimiento endoscópico. Todas las preparaciones histológicas fueron revisadas por un único patólogo para evitar diferencias inter-observador.

Definimos como duración del seguimiento endoscópico a los meses transcurridos entre la resección del carcinoma colo-rectal y la fecha de la última colonoscopia de control. Detallamos el número de endoscopias de control efectuadas a cada paciente, así como su media y desviación típica. Cuantificamos el periodo de tiempo (meses), su mediana y rango, transcurrido entre la extirpación del tumor y la práctica de las sucesivas endoscopias de seguimiento.

Consideramos como lesiones neoplásicas metacrónicas a los carcinomas y adenomas, excluyendo los pólipos hiperplásicos. Denominamos cáncer metacrónico a la lesión tumoral maligna, confirmada histológicamente, que no asienta en la anastomosis y que se objetiva al menos 12 meses después de la operación quirúrgica ${ }^{14}$. No incluimos a la recidiva tumoral como una lesión metacrónica, por lo que no será objeto de nuestro estudio. 
Detallamos las características de los cánceres metacrónicos, referentes a:

- Número de tumores diagnosticados

- Localización anatómica

- Número de la colonoscopia de control en que se objetivó la lesión

- Periodo de tiempo transcurrido entre la resección del primer carcinoma y el del tumor metacrónico

Registramos el número de pacientes que presentaron adenomas metacrónicos, tanto simples como avanzados, a lo largo del seguimiento endoscópico. Consideramos adenoma avanzado al pólipo que presenta componente velloso o tamaño superior a $10 \mathrm{~mm}$ o displasia de alto grado ${ }^{14}$. Se estudia la posible diferencia de incidencia de estas lesiones entre los dos hospitales que forman nuestra serie.

Analizamos la frecuencia con la que los adenomas fueron detectados en cada una de las colonoscopias de control que se practicaron. Adicionalmente, se determinó la frecuencia de adenomas metacrónicos diagnosticados en los casos en los que la primera colonoscopia se efectuó a los 12 meses del diagnóstico, registrándose la proporción de lesiones menores de $5 \mathrm{~mm}$.

Centrándonos en la primera exploración que detecta lesiones metacrónicas, se estudian las siguientes características de los adenomas:

- Número de pólipos: lesión única, de 2 a 5 adenomas y más de 10

- Tamaño del mayor adenoma encontrado $<5 \mathrm{~mm}$, entre 5 y $10 \mathrm{~mm}$ y $>10$ $\mathrm{mm}$

- Localización con respecto al ángulo esplénico: proximal, distal o ambas

- Tipo histológico del adenoma: tubular, tubulo-velloso, velloso y adenoma serrado

Registramos el periodo medio de tiempo transcurrido entre la resección del cáncer inicial y el diagnóstico del primer adenoma metacrónico, tanto simple como avanzado.
Para el estudio estadístico empleamos los tests de Kolmogorov-Smirnoff, Fisher y Chi cuadrado. Consideramos significativos los valores de $\mathrm{p}<0,05$. Los resultados se expresan como media aritmética y desviación típica cuando las variables siguieron una distribución normal y como mediana y rango en el resto.

\section{RESULTADOS}

La mediana de duración del seguimiento mediante colonoscopias completas fue de 48 meses (12-112). Al tratarse de un estudio retrospectivo, el momento de la realización de las sucesivas endoscopias de control no estaba previamente establecido. En la figura 1 se representa el tiempo tras la cirugía, expresado en medianas, en el que se efectuaron las sucesivas colonoscopias.

La media de colonoscopias de control practicadas fue de $2,74 \pm 1,47$. El número de exploraciones realizadas a cada paciente se representa en la figura 2.

A lo largo del seguimiento se registraron 7 cánceres metacrónicos, todos ellos adenocarcinomas, lo que supone una incidencia del 1,8\% sobre el total de los pacientes. Registramos 4 casos de pólipo malignizado, estadio I, resecados endoscópicamente. Dos pacientes fueron reintervenidos, uno con estadio III-C y otro IV. El último caso recibió quimioterapia, no pudiendo precisarse su estadio pTNM.

Sus localizaciones fueron: 2 casos en recto, 2 en sigma, 1 en colon descendente, 1 en colon transverso y 1 colon en ascendente-ciego.

El diagnóstico se efectuó en la primera colonoscopia de control en 3 casos, en la segunda endoscopia en 1 paciente y en la cuarta exploración en 3 . El periodo de latencia medio entre el diagnóstico del primer cáncer y el carcinoma metacrónico tuvo una mediana de 24 meses (rango: 13-54). 


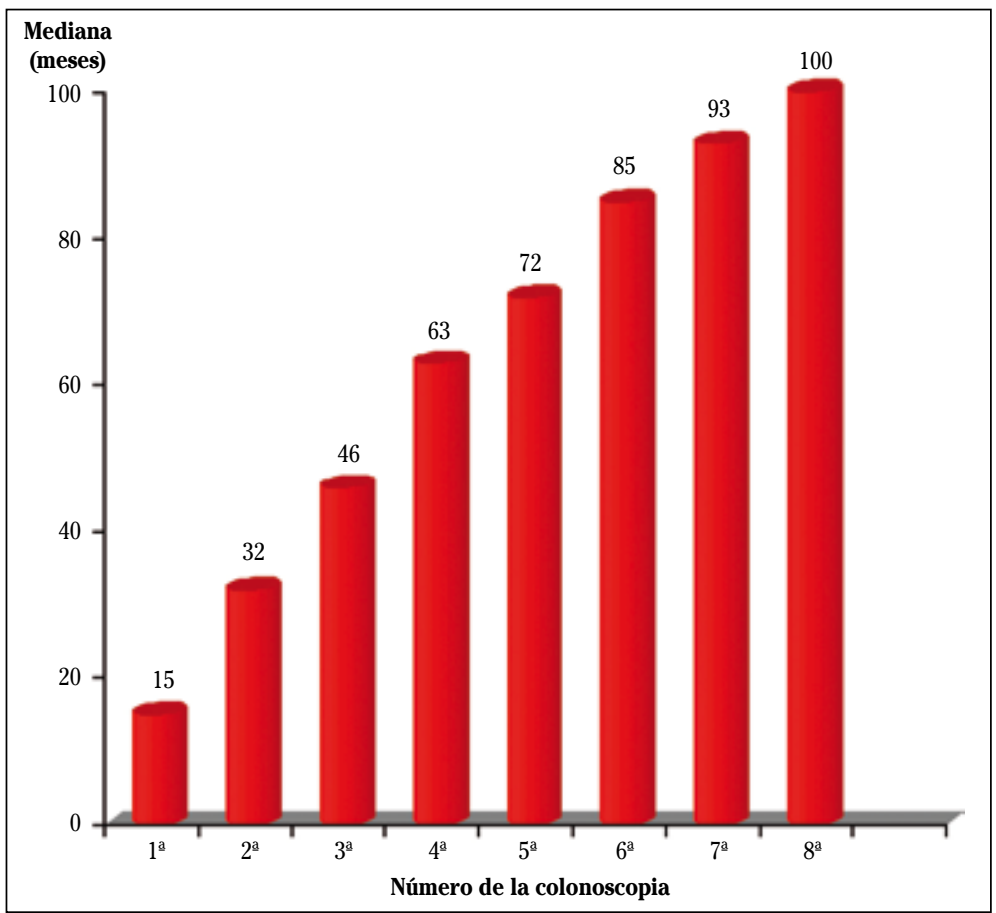

Figura 1. Tiempo tras la cirugía de las sucesivas exploraciones de control.

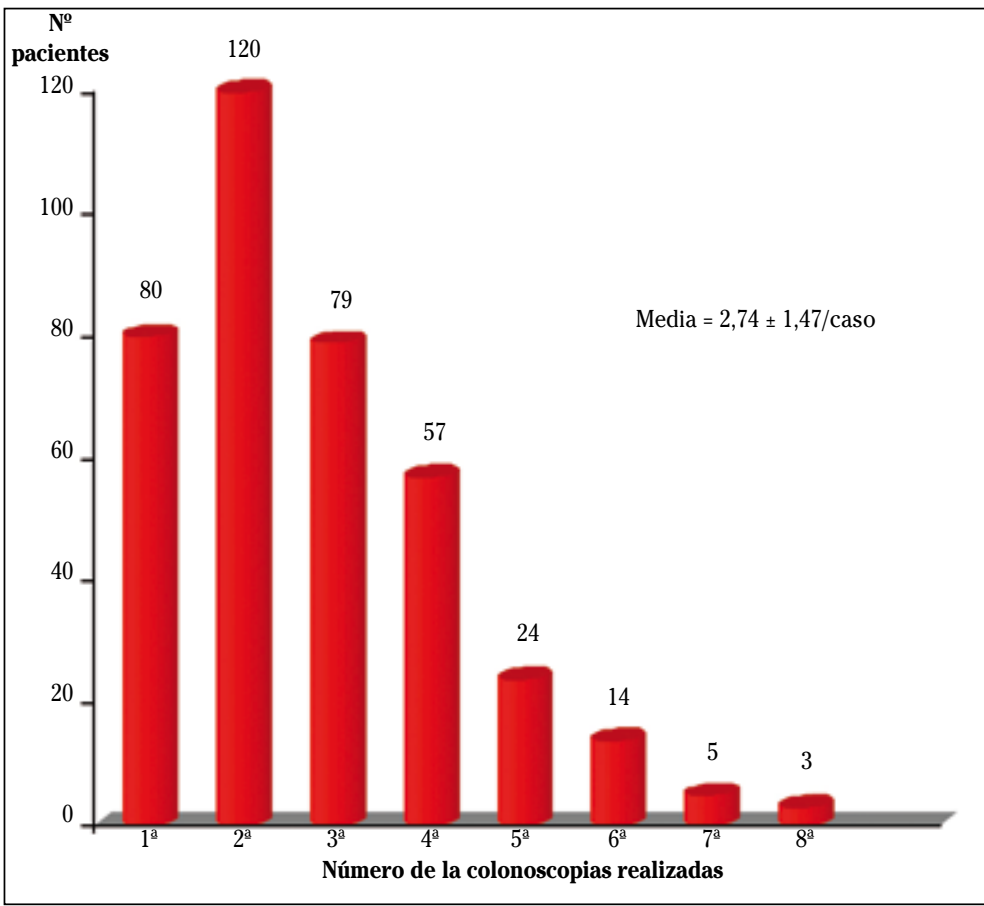

Figura 2. Número de endoscopias de control efectuadas a cada paciente. 
En 162 de los 382 pacientes $(42,4 \%)$ se diagnosticaron adenomas durante el seguimiento. La incidencia de adenomas fue muy similar entre nuestros dos hospitales:
$42,1 \%$ frente a $43,2 \%(\mathrm{p}=0,88)$ (Fig. 3$)$. En la figura 4 se representa el número de la colonoscopia de control en la que se diagnostica el primer adenoma metacrónico.

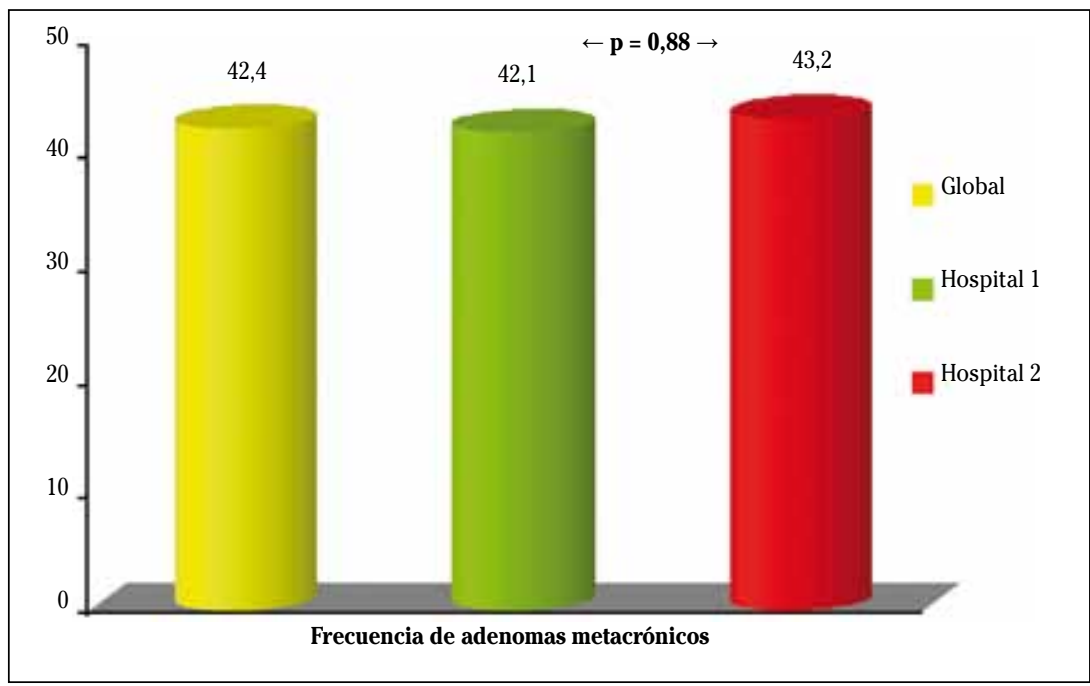

Figura 3. Adenomas metacrónicos diagnosticados: frecuencia global y en los dos hospitales de nuestra serie.

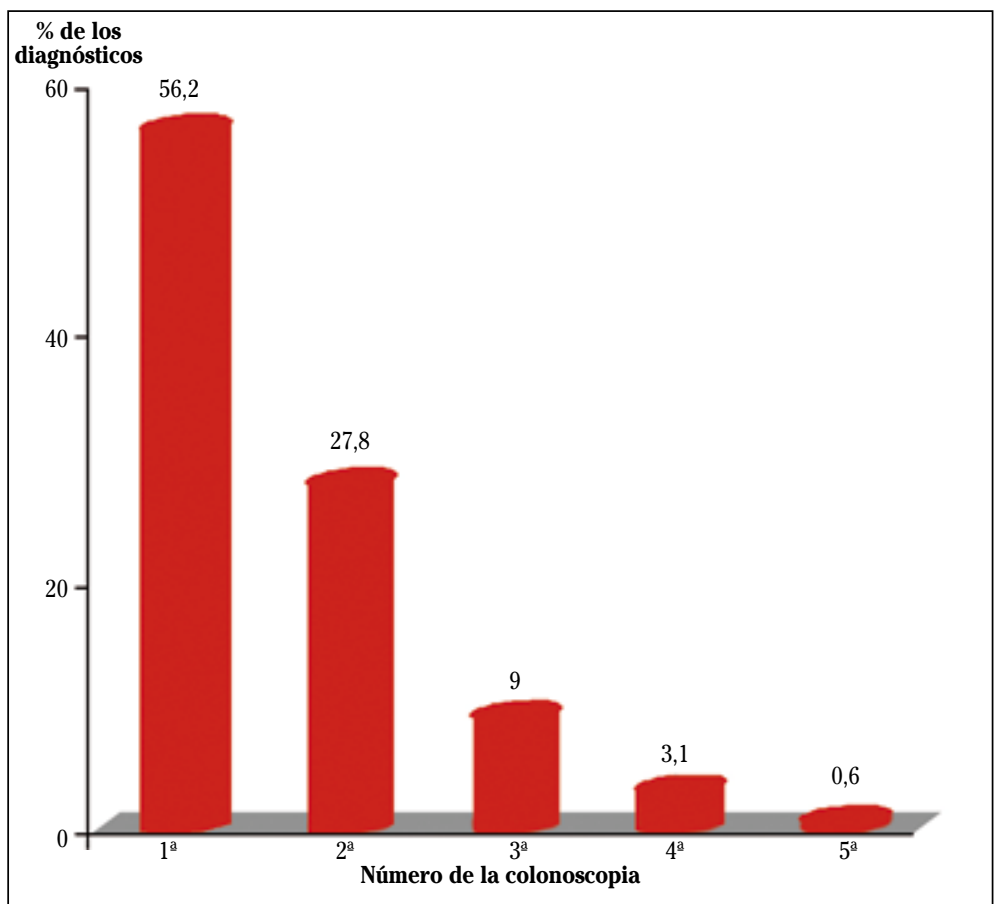

Figura 4. Número de endoscopia de control en la que se diagnostica la primera lesión metacrónica. 
En los 164 pacientes en los que la primera exploración de control se efectuó a los 12 meses, la incidencia de adenomas fue del $24 \%$. En estos casos, el tamaño de la lesión fue $<5 \mathrm{~mm}$ en un $86,5 \%$ frente a un $72,2 \%$ de lesiones $<5 \mathrm{~mm}$ cuando el diagnóstico se realizó más tardíamente $(\mathrm{p}=0,10)$ (Fig. 5).

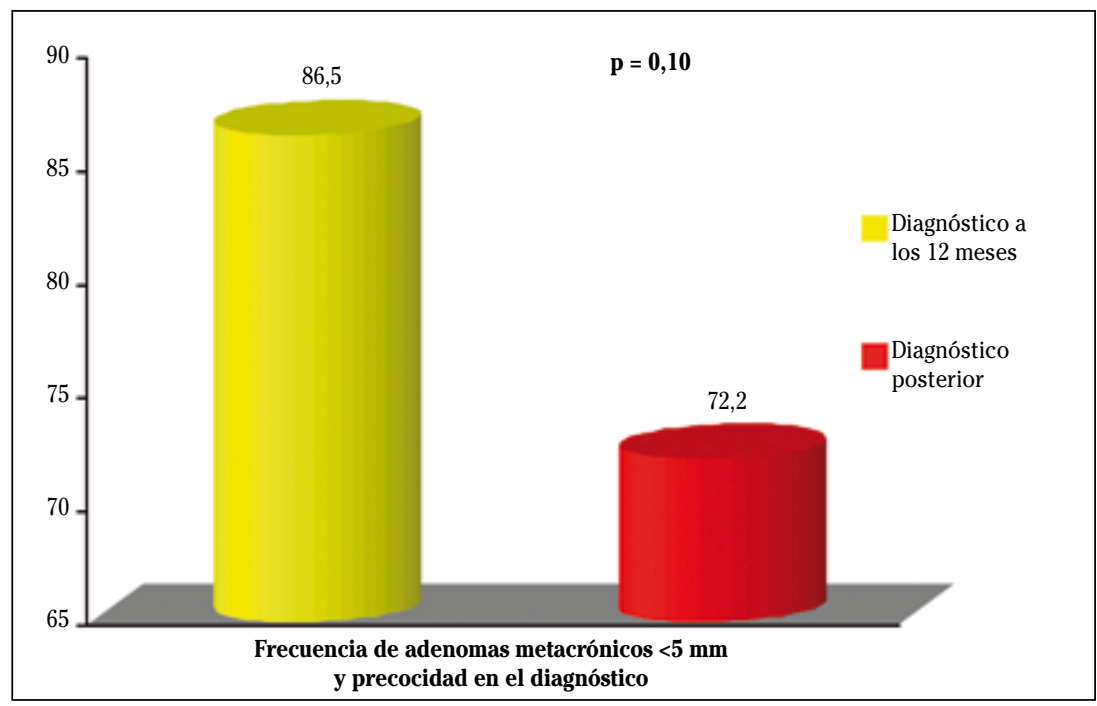

Figura 5. Frecuencia de adenomas metacrónicos menores de $5 \mathrm{~mm}$ entre los casos de diagnóstico precoz (12 meses) y el resto.

Detectamos adenomas avanzados en $24 / 382$ enfermos, lo que supone el $6,3 \%$ del global de pacientes y el $14,8 \%$ de los que desarrollan adenomas.

En la tabla 1 se resumen las características: localización, número y tamaño de los primeros adenomas metacrónicos diagnosticados.

Tabla 1. Características de los primeros adenomas metacrónicos diagnosticados.

\begin{tabular}{|c|c|c|c|}
\hline Localización & $\begin{array}{l}\text { Distal } \\
44,5 \%\end{array}$ & $\begin{array}{c}\text { Proximal } \\
39,5 \%\end{array}$ & $\begin{array}{c}\text { Ambas } \\
16 \%\end{array}$ \\
\hline \multirow{2}{*}{ Número } & Único & $2-5$ & $6-10$ \\
\hline & $60,8 \%$ & $38,5 \%$ & $0,7 \%$ \\
\hline \multirow{2}{*}{ Tamaño } & $<5 \mathrm{~mm}$ & $5-10 \mathrm{~mm}$ & $>10 \mathrm{~mm}$ \\
\hline & $68,5 \%$ & $26,2 \%$ & $5,3 \%$ \\
\hline
\end{tabular}

Por lo que respecta a la histología y, considerando la lesión más avanzada en los casos de pólipos múltiples, la distribución fue: adenoma tubular $=83,6 \%$, túbulovelloso $=9,3 \%$, velloso $=1,4 \%$ y adenoma serrado $=5,7 \%$.

El periodo de tiempo transcurrido entre la cirugía del cáncer inicial y el diagnóstico del primer adenoma metacrónico ha registrado una mediana de 21 meses (12-112).

El intervalo diagnóstico del primer adenoma metacrónico avanzado presentó una mediana de 35 meses (12-112).

\section{DISCUSIÓN}

La incidencia de adenomas y carcinomas colo-rectales metacrónicos muestra unas amplias variaciones en las diversas series publicadas, probablemente por los diferentes criterios utilizados a la hora de definir la lesión metacrónica, los distintos periodos de seguimiento ${ }^{13}$, así como de las 
diversas técnicas de exploración aplicadas tras la extirpación quirúrgica del cáncer colo-rectal ${ }^{8,15,16}$.

Como la mayoría de las publicaciones ${ }^{15-18}$, nuestro trabajo se basa en un estudio retrospectivo. Por este motivo los pacientes no fueron sometidos a revisión endoscópica en periodos de tiempo fijos y pre-establecidos, sino que presentaron variaciones en cuanto al momento en el que se efectuaron las colonoscopias y a su frecuencia. La duración del control evolutivo ha sido variable, presentando una mediana de 48 meses tras el momento del diagnóstico inicial. Nuestro periodo de seguimiento se sitúa en la zona media de los estudios publicados, que varían entre los 12 meses y los 9 años ${ }^{8,9,19,20}$.

Aunque en unos pocos pacientes se efectuaron hasta 8 colonoscopias a lo largo del control evolutivo, el número más frecuente de exploraciones ha oscilado entre 1 y 3 , con una media de 2,74 endoscopias por caso. Esta frecuencia de exploraciones es más alta que la de otras series, que tan solo las realizan en una ocasión ${ }^{13,19,20}$, aunque inferior a la de un estudio italiano del año 2000, que alcanza una mediana de 4,6 colonoscopias por paciente ${ }^{9}$.

En cuanto al momento evolutivo en el que se realizaron las sucesivas endoscopias, los valores obtenidos muestran una distribución bastante regular, con una mediana de 15 meses para el primer control y unas medianas de $32,46,63,71,83,93$ y 100 meses para las siguientes exploraciones, lo que expresa una buena progresión en los sucesivos intervalos medios de seguimiento. Este control evolutivo nos ha permitido diagnosticar una serie de lesiones neoplásicas metacrónicas.

No hemos incluido a las recidivas tumorales como lesiones metacrónicas. Dichas recidivas se consideran como una progresión loco-regional del tumor inicial, a pesar de que la cirugía se efectuase con intención curativa y no como nuevas lesiones neoplásicas $^{21}$. Se define como cáncer colo-rectal metacrónico al tumor que se presenta tras la resección de todas las lesiones neoplásicas iniciales ${ }^{22,23}$. Este segundo tumor no debe ser una recidiva en la anastomosis quirúrgica o un depósito metastático del anterior $^{24}$.

Se ha discutido el periodo de tiempo que ha tenido que transcurrir entre el diagnóstico de los dos tumores, para poder considerar al segundo como independiente y por tanto metacrónico. Aunque se han propuesto plazos entre 6 meses y 3 años ${ }^{13,15,19,25,26}$, al igual que otros autores hemos establecido el periodo de un año tras el primer diagnóstico, para reducir el riesgo de considerar como metacrónica a una lesión sincrónica no advertida ${ }^{13,25,26}$. El examen endoscópico de la totalidad del colon se sigue considerando como el "patrón oro" para el diagnóstico de los tumores $\operatorname{colónicos}^{27,28}$. No obstante, se ha estimado que la colonoscopia puede llegar a omitir hasta un 5,5-5,9\% de los cánceres de localización proximal, descendiendo al 2,1-2,3\% en el distal ${ }^{29}$. Por ello cabe la posibilidad de que algún tumor aparentemente metacrónico pueda corresponder a lesiones sincrónicas no diagnosticadas previamente ${ }^{30}$. Se ha estimado que la duración de la secuencia adenoma-carcinoma pueda ser de unos 10 años ${ }^{15}$, pero se desconoce si este periodo de tiempo puede aplicarse a los cánceres metacrónicos ${ }^{15}$, siendo habitual encontrar una pequeña proporción de pacientes que presentan un carcinoma metacrónico en los cinco primeros años tras la resección de las lesiones iniciales.

Nuestra frecuencia de cáncer metacrónico ha sido del $1,8 \%$, cifra concordante con la de la mayoría de las series, que fluctúan entre el 1 y el 5\% $\%^{8-12,31}$ y muy similar a la de un reciente estudio que incluye 19 hospitales españoles, que registraba una incidencia del 1,9 \% ${ }^{13}$.

La frecuencia del hallazgo de cánceres metacrónicos puede estar relacionada con el tipo de exploraciones empleadas para su diagnóstico, considerándose como la más adecuada la práctica de colonoscopias completas $^{32}$, como en nuestra serie. Por otra parte, y como es lógico, el porcentaje de cánceres metacrónicos está influido por la duración del seguimiento ${ }^{19}$, por lo que los valores más altos suelen corresponder 
a controles evolutivos más prolongados en el tiempo ${ }^{8}$. En un estudio francés de reciente publicación, se confirma el progresivo incremento en la incidencia de cáncer metacrónico a lo largo del seguimiento, registrándose un tasa acumulativa del $1,8 \%$ a los 5 años, que asciende al $3,4 \%$ a los 10 años y al $7,2 \%$ a los 20 años $^{33}$. También disponemos de un estudio evolutivo a muy largo plazo, donde se alcanza un 30\% de cáncer metacrónico a los 41 años de seguimiento ${ }^{34}$.

Como en otros trabajos ${ }^{35}$, la localización del cáncer metacrónico ha sido predominantemente distal, con sólo dos tumores proximales al ángulo esplénico.

El tiempo transcurrido entre los diagnósticos del primer y segundo carcinoma puede variar según el programa de seguimiento endoscópico que se haya establecido, ya que en muchas ocasiones el cáncer metacrónico es un hallazgo exploratorio en un paciente asintomático ${ }^{16}$. En nuestra serie la mayoría de los cánceres no se diagnosticaron en la primera colonoscopia de control, lo que apoya que no se trataba de tumores previos inadvertidos, siendo la mediana del intervalo diagnóstico de 24 meses, inferior a los 71 meses de otros autores $^{10}$.

Independientemente del estudio de posibles recidivas tumorales, el principal objetivo de realizar exploraciones de control tras la resección del tumor inicial es de poder diagnosticar lesiones metacrónicas en fases menos avanzadas, que puedan beneficiarse de tratamientos más eficaces. En nuestra serie conseguimos detectar cuatro de los siete cánceres metacrónicos en fase de pólipo malignizado, pudiéndose efectuar un tratamiento endoscópico curativo.

Con respecto a los adenomas metacrónicos, nuestros pacientes presentaron una alta incidencia $(42,4 \%)$, que se sitúa en el límite superior de las series publicadas, con unas frecuencias del $25-48 \%^{20,31}$, superando al $21,5 \%$ de lesiones registradas en un estudio cooperativo nacional ${ }^{13}$. La explicación a esta elevada incidencia puede basarse en que nuestro seguimiento post-operatorio se ha realizado en todos los casos mediante colonoscopias completas. El hecho de que la incidencia de adenomas metacrónicos haya sido prácticamente idéntica en los dos hospitales que forman nuestra casuística y que presentan unas características asistenciales diferentes, confiere una mayor credibilidad a nuestros resultados y sugiere que la frecuencia real de lesiones metacrónicas haya podido estar infravalorada en otros estudios.

De los pacientes que desarrollaron adenomas metacrónicos, en un 56,2\% de los casos las lesiones se objetivaron en la primera endoscopia de control, siendo cada vez menos frecuente el diagnóstico de nuevos casos en las siguientes exploraciones que se realizaron, con solo un $3 \%$ de nuevos diagnósticos a partir de la tercera exploración.

Consideramos destacable el que en uno de cada cuatro pacientes que desarrollan adenomas, esta lesión es ya detectable a los 12 meses de la resección. Estos adenomas "precoces" parecen ser auténticamente metacrónicos, ya que su tamaño es inferior al del resto de lesiones diagnosticadas más tardíamente. Si se tratase de lesiones previas omitidas, lógicamente estarían más evolucionadas y su tamaño hubiera sido mayor. Nuestros resultados parecen indicar que, en el paciente predispuesto a presentar adenomas metacrónicos, las lesiones se desarrollan de forma precoz tras la resección inicial. Por ello la mayoría de los adenomas se diagnostican ya en la primera o segunda endoscopia, siendo escasa la probabilidad de nuevos diagnósticos en los sucesivos controles.

La localización dentro del colon de los adenomas neoplásicos metacrónicos se ha descrito como preferentemente distal ${ }^{36-38}$. No obstante, debemos considerar que se ha referido una mayor proporción de lesiones metacrónicas proximales si se compara con la localización de las lesiones sincrónicas iniciales ${ }^{39,40}$, manteniéndose esta tendencia hacia una mayor localización proximal más frecuente en los adenomas de tercera generación ${ }^{39}$. En nuestra serie hay que resaltar que la mayoría de los pacientes presentaban alguna lesión proximal al ángulo esplénico. Esta localización fue exclusivamente proxi- 
mal en un $39,5 \%$ y con menor frecuencia las lesiones asentaron en ambos segmentos (16\%). La consecuencia práctica de nuestros resultados es que, independientemente de la localización de las lesiones iniciales, el seguimiento debe realizarse mediante estudio endoscópico completo del colon, ya que más de la mitad de los casos presentan algún adenoma proximal.

$\mathrm{Al}$ ser limitado el tiempo de seguimiento, era esperable que el número de adenomas por paciente y el tamaño de los mismos sea reducido en las lesiones metacrónicas ${ }^{41,42}$. Como en otros trabajos, el primer adenoma metacrónico diagnosticado fue mayoritariamente único, siendo excepcionales los casos que superaban los cinco pó$\operatorname{lipos}^{13}$. Más de dos tercios de los adenomas fueron menores de $5 \mathrm{~mm}$, cifra superior a la del ya mencionado estudio multicéntrico nacional ${ }^{13}$ que describe una frecuencia del $56,2 \%$. Por último, tan sólo un $5 \%$ del total de las lesiones metacrónicas rebasaron los $10 \mathrm{~mm}$. No se conoce con precisión el ritmo de crecimiento de los adenomas, que puede ser no lineal ${ }^{43}$, disponiendo tan sólo de algún dato referente a los adenomas sincrónicos. Se ha estimado un crecimiento diametral aproximado de $0,5 \mathrm{~mm} /$ $\mathrm{anno}^{43}$, aunque también se ha descrito en un $10 \%$ de los adenomas un crecimiento mucho más rápido, de $2-4 \mathrm{~mm} / \mathrm{año}^{44} \mathrm{e}$ incluso, de modo excepcional, de $10 \mathrm{~mm}$ en un sólo $a n ̃ o^{45}$. Teniendo en cuenta estos datos, es probable que nuestros escasos pólipos de tamaño superior a $10 \mathrm{~mm}$ correspondan en realidad a casos aislados de adenomas previos no visualizados en la colonoscopia que diagnóstico el cáncer inicial. Esta omisión endoscópica de los pólipos es un dato bien conocido, con un rango que oscila entre el 6 y el $27 \%$ según el tamaño de la lesión ${ }^{46-48}$.

La clasificación histológica de los adenomas metacrónicos ha sido similar a la referida por otros autores ${ }^{36}$, con un claro predominio de las formas tubulares. Es destacable el hallazgo de un 5,7\% de adenomas serrados metacrónicos, cifra superior a la habitualmente referida para las lesiones sincrónicas ${ }^{49}$.
Por lo que respecta a los adenomas metacrónicos avanzados, hemos registrado una frecuencia global del $6,3 \%$, idéntica a la de otra serie publicada en el año $2003^{50}$. Al ser el adenoma avanzado una lesión más evolucionada, su incidencia en la primera colonoscopia de control ha sido significativamente menor con respecto al resto de los adenomas. Aunque de modo sorprendente algún autor ha referido un menor periodo de tiempo hasta el diagnóstico de los adenomas avanzados que de los no avanzados $^{51}$, consideramos que nuestros resultados son más fácilmente explicables, al registrar un intervalo diagnóstico más prolongado para las lesiones avanzadas: 35 meses, que para el resto (21 meses).

A modo de conclusión, podemos destacar que el seguimiento que hemos realizado a nuestros pacientes mediante colonoscopias completas nos ha permitido aplicar un tratamiento teóricamente curativo en la mayoría de los carcinomas metacrónicos diagnosticados. Por lo que respecta a los adenomas, la alta frecuencia con la que se han presentado y su elevada proporción de localización proximal hacen necesario un seguimiento endoscópico que alcance el ciego. En la actualidad, todavía no disponemos de una pauta de seguimiento ideal que precise el momento de inicio, la frecuencia y la duración del seguimiento endoscópico tras la resección del CCR con intención curativa. Según nuestros resultados, la primera colonoscopia de control debiera efectuarse a los doce meses de la cirugía. Igualmente, los pacientes que tras 3 endoscopias consecutivas no mostraran adenomas metacrónicos, presentarían un riesgo muy reducido de desarrollarlos posteriormente, por lo que a partir de ese momento precisarían un control endoscópico mucho menos estricto.

\section{BIBLIOGRAFÍA}

1. Weitz J, Koch M, Debus J, Höhler T, Galle PR, Büchler MW. Colorectal cancer. Lancet 2005; 365: 153-165.

2. Russo MW, Wei JT, Thiny MT, Gangarosa LM, Brown A, Ringel Y et al. Digestive and liver diseases statistics. Gastroenterology 2004; 126: 1448-1453. 
3. Ardanaz E, Moreno-Iribas C, Pérez de Rada ME, EZPonda C, Floristán Y, Navaridas $\mathrm{N}$ et al. Incidencia y mortalidad por cáncer en Navarra, 1998-2002. Evolución en los últimos 30 años. An Sist Sanit Navar 2007; 30: 245-270.

4. Rodrigo L, Riestra S. Dieta y cáncer de colon. Rev Esp Enferm Dig 2007; 99: 183-189.

5. Castells A, Marzo M, Bellas B, Amador FJ, Lanas A, MASCORT JJ et al. Guía de práctica clínica en prevención del cáncer colo-rectal. Gastroenterol Hepatol 2004; 27: 573-634.

6. Piñol V, Andreu M, Castells A, Paya A, Bessa X, JOVER R. Synchronous colorectal neoplasms in patients with colorectal cancer: predisposing individual and familial factors. Dis Col Rectum 2004; 47: 1192-2000.

7. Borda A, Martínez Peñuela J, Prieto C, Muñoz M, Carretero C, Borda F. Estudio de la frecuencia, distribución y rendimiento diagnóstico en las lesiones neoplásicas sincrónicas del carcinoma colo-rectal. An Sist Sanit Navar 2008; 31: 43-52.

8. Shitoh K, Konishi F, Miyakura Y, Togashi K, OKaмото T, NAGA H. Microsatellite instability as a marker in predicting metachronous multiple colorectal carcinomas after surgery: a cohort-like study. Dis Colon Rectum 2002; 45 : 329-333.

9. Stigliano V, Fracasso P, Grassi A, Lapenta R, CiTARDA F, TOMASEll $\mathrm{G}$ et al. Endoscopic followup in resected colorectal cancer patients. $\mathrm{J}$ Exp Clin Cancer Res 2000; 19: 145-148.

10. LAN YT, LiN JK, Li AF, Lin TC, Chen WS, JiAng JK et al. Metachronous colorectal cancer: Necessity of post-operative colonoscopic surveillance. Int J Colorectal Dis 2005; 20: 121-125.

11. Chen HS, Sheen-Chen SM. Synchronous and «early» metachronous colorectal adenocarcinoma: analysis of prognosis and current trends. Dis Colon Rectum 2000; 43: 10931099.

12. Tsantilas D, Ntinas A, Petras P, Zambas N, Al Mogrambi S, Frangandreas G et al. Metachronous colorectal adenocarcinomas. Tech Coloproctol 2004; 8: 1: 202-204.

13. Gschwantler M, Kriwanek S, Langner E, Göritzer B, SchrutKa-Kölbl C, Brownstone E et al Highgrade dysplasia and invasive carcinoma in colorectal adenomas: a multivariate analysis of the impact of adenoma and patient characteristics. Eur J Gastroenterol Hepatol 2002; 14: 183-188.

14. Ballesté B, Bessa X, Piñol V, Castellví-Bel S, Castells A, Alenda $C$ et al. Detection of meta- chronous neoplasm in the colorectal cancer patients. Identification of risk factors. Dis Colon Rectum 2007; 50: 971-980.

15. Fajobi O, Yiu CY, Sen-Gupta SB, Boulos PB. Metachronous colorectal cancers. Br J Surg 1998; 85: 897-901.

16. Yamazaki T, TaKiI Y, OKamoto $\mathrm{H}$, SaKai $\mathrm{Y}$, HataKeYAMA $\mathrm{K}$. What is the risk factor for metachronous colorectal carcinoma? Dis Colon Rectum 1997; 40: 935-938.

17. NaH BK, Kim SM, Lee YS, Yang HW, Seo SW, Sung JK et al. Pattern of metachronous adenoma after colorectal cancer surgery. Korean $\mathrm{J}$ Gastroenterol 2004; 44: 212-216.

18. Kulling D, Christ AD, Karaaslan N, Fried M, BAUERFEIND P. The presence of more than two index adenomas is the strongest predictor of metachronous colon adenomas. Swiss Med Wkly 2002; 132: 139-142.

19. Cali RL, Pitsch RM, Thorson AG, Watson P, TAPIA P, BLATChFord GJ et al. Cumulative incidence of metachronous colorectal cancer. Dis Colon Rectum 1993; 36: 388-393.

20. Сно JH, LeE SK, Kiм TI, Kiм WH. Characteristics and risk factors of synchronous and metachronous polyp in colorectal cancer. Korean J Gastroenterol 2004; 43: 168.

21. Rodríguez-Moranta F, Saló J, Arcusa A, Boadas J, Piñol V, Bessa X et al. Postoperative surveillance in patients with colorectal cancer who have undergone curative resection: a prospective, multicenter, randomized, controlled trial. J Clin Oncol 2006; 24: 386-393.

22. Agrez MV, Ready R, Ilstrup D, Beart RW. Metachronous colorectal malignancies. Dis Colon Rectum 1982; 25: 569-574.

23. Finan PJ, Ritchie JK, Hawley PR. Synchronous and "early" metachronous carcinomas of the colon and rectum. Br J Surg 1987; 74: 945-947.

24. WarRen S, GATES O. Multiple primary malignant tumors: a survey of the literature and a statistical study. Am J Cancer 1932 ; 16: 1358-1414.

25. Kaibara N, Koga S, Kinnal D. Synchronous and metachronous malignancies of the colon and rectum in Japan with special reference to a coexisting early cancer. Cancer $1984 ; 54$ : 1870-1874.

26. MacGivillivray DC, Swartz SE, Robinson AM, CRuess DF, Smith LE. Adenocarcinoma of the colon and rectum in patiens less than 40 years of age. Surg Gynecol Obstet 1991; 172: 1-7.

27. Blachar A, Sosna J. CT colonography (virtual colonoscopy): technique, indications and performance. Digestion 2007; 76: 34-41. 
28. Macken E, Moreels T, Pelckmans M, Baert D, ReyNAERT H,DELOOZE D et al Quality assurance and recommendations for quality assessment of screening colonoscopy in Belgium. Acta Gastroenterol Bel 2009; 72: 17-25.

29. Bressler B, Paszat LF, Chen Z, Rothwell DM, VinDEN C, RABENECH L. Rates of new or missed colorectal cancers after colonoscopy and their risk factors: a population-based analysis. Gastroenterology 2007; 132: 96-102.

30. Green RJ, Metlay JP, Propert K, Catalano PJ, Macdonald JS, MAYer RJ et al. Surveillance for second primary colorectal cancer after adjuvant chemotherapy: an analysis of intergroup 0089. Ann Intern Med 2002; 136: 261-269.

31. Fukutumi Y, Moriwaki H, Nagase S, Tajika M, Naito T, Miwa $Y$ et al. Metachronous colon tumors: risk factors and rationale for the surveillance colonoscopy after initial polypectomy. J Cancer Res Clin Oncol 2002; 128: 569-574.

32. Rajaratnam SG, Dennett ER. Development of metachronous neoplasms alter colorrectal cancer resection: absence of synchronous neoplams predicts a lower risk. N Z Med J 2009; 122: 61-66.

33. Bouvier AM, Latournerie M, Jooste V, Lepage C, Cоттет V, FaIVRE J. The lifelong risk of metachronous colorectal cancer justifies longterm colonoscopic follow-up. Eur J Cancer 2008; 44: 522-527.

34. Bulow S, Svendsen LB, Mellemgaard A. Metachronous colorectal carcinoma. Br J Surg 1990; 77: 502-505.

35. Cerdán J, Torres-Melero J, Díez M, Balsa T, BaLIBREA JL. Adenocarcinomas colo-rectales metacrónicos. Rev Esp Enferm Digest 1996; 88: 655-659.

36. Nusko G, Hahn EG, Mansmann U. Characteristics of metachronous colorrectal adenomas found during long-term follow-up: analysis of four subsequent generations of adenoma recurrence. Scand J Gastroenterol 2009; 44: 736-744.

37. Cappell MS, Forde KA. Spatial clustering of multiple hyperplastic, adenomatous, and malignant colonic polyps in individual patients. Dis Colon Rectum 1989; 32: 641-652.

38. Olsen HW, Lawrence WA, SNook CW, Mutch WM. Review of recurrent polyps and cancer in 500 patients with initial colonoscopy for polyps. Dis Colon Rectum 1988; 31: 222-227.

39. Nusko G, Mansmann U, Wiest G, Brueckl W, KirCHNER T, HAHN EG et al. Right-sided shift found in metachronous colorectal adenomas. Endoscopy 2001; 33: 574-579.
40. Evers BM, Mullins RJ, Mathews TH, Broghamer WL, PolK HC. Multiple adenocarcinomas of the colon and rectum. An analysis of incidences and current trends. Dis Colon Rectum 1988; 31: 518-522.

41. Griffioen G, Bosman FT, Verspaget HW, Sier KF, Biemond I, LAmers CB. Comparative evaluation of carcinoembryonic antigen, secretory component, and mucins in index and metachronous adenomas of the colorectum. Gastroenterology 1991; 101: 919-926.

42. Hofstad B, Vatn MH, Andersen SN, Huttfeldt HS, Rognum T, LARSEN $\mathrm{S}$ et al. Growth of colorectal polyps: redetection and evaluation of unresected polyps for a period of three years. Gut 1996; 39: 449-456.

43. TADA M, Misaki F, DaWaI K. Growth rates of colorectal carcinoma and adenoma by roentgenologic follow-up observations. Gastroenterol Jpn 1984; 19: 550-555.

44. Bersentes K, Fennerty MB, Sampliner RE, GaREWAL HS. Lack of spontaneus regression of tubular adenomas in two years of follow-up. Am J Gastroenterol 1997; 92: 1117-1120.

45. Hofstad B, Tatn M, Larsen S, Osnes M. Growth of colorectal polyps: recovery and evaluation of unresected polyps of less than 10 $\mathrm{mm}, 1$ year after detection. Scand J Gastroenterol 1994; 29: 640-645.

46. Heresbach D, Barrioz T, Lapalus MG, Coumaros D, Bauret P, Potier P et al. Miss rate for colorectal neoplastic polyps: a prospective multicenter study of back-to-back video colonoscopies. Endoscopy 2008; 40: 284-290.

47. Van Rijn JC, Reitsma JB, Stoker J, Bossuyt PM, Van Deventer SJ, Dekrer E. Polyp miss rate determined by tandem colonoscopy: a systematic review. Am J Gastroenterol 2006; 101: 343-350.

48. Rex DK, Cutler CS, Lemmel CT, Rahmani EY, Clark DW et al. Colonoscopic missrates of adenomas determined by back-to-back colonoscopies. Gastroenterology 1997; 112: 24-28.

49. Rüschoff J, Aust D, HaRTmann A. Colorectal serrated adenoma: diagnostic criteria and clinical implications. Verh Dtsch Ges Pathol 2007; 91: 119-125.

50. SAINI SD, Kim HM, Schoenfeld P. Incidence of advanced adenomas at surveillance colonoscopy in patients with a personal history of colon adenomas: a meta-analysis and systematic review. Gastrointest Endosc 2006; 64: 614-626.

51. McFall MR, Woods WG, Miles WF. Colonoscopic surveillance after curative colorectal resection: results of an empirical surveillance programme. Colorectal Dis 2003; 5: 131-132. 